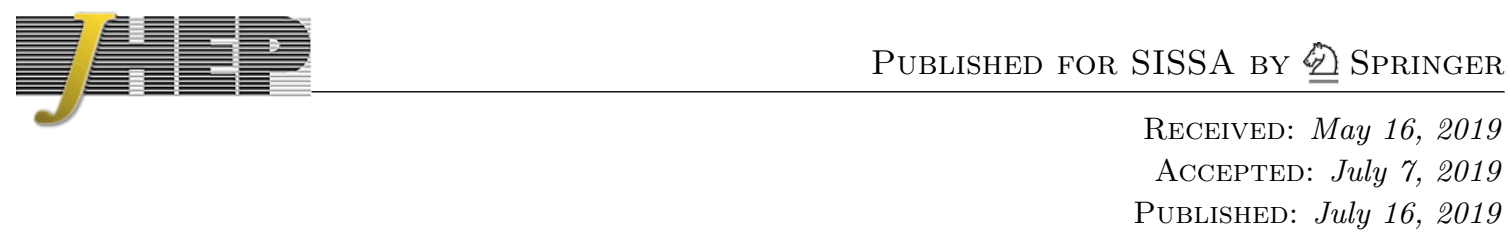

\title{
More on Wilson loops for two touching circles
}

\author{
Harald Dorn \\ Institut für Physik und IRIS Adlershof, Humboldt-Universität zu Berlin, \\ Zum Großen Windkanal 6, D-12489 Berlin, Germany \\ E-mail: dorn@physik.hu-berlin.de
}

ABSTRACT: We calculate both at leading weak and strong coupling the renormalised Maldacena-Wilson loop for contours formed by consecutive passage of two touching circles. At the touching point both circles should have the same normal direction but form cusps of non-zero opening angle $\alpha$. Particular emphasis is put on the behaviour in the limit $\alpha \rightarrow 0$ and its comparison with the spiky situation studied in a previous paper, where $\alpha$ was set to zero before renormalisation.

Keywords: Wilson, 't Hooft and Polyakov loops, AdS-CFT Correspondence, Renormalization Regularization and Renormalons

ArXiv EPrint: 1905.01101 


\section{Contents}

1 Introduction 1

2 Lowest order at weak coupling in $\mathcal{N}=4$ SYM 3

$\begin{array}{lll}3 & \text { The case of equal radii } & 6\end{array}$

$\begin{array}{lll}4 & \text { Holographic evaluation at strong coupling } & 7\end{array}$

5 Summary and discussion $\quad 12$

A Evaluation of the integral $I_{12}^{(2), \text { rest }} \quad 13$

B $\epsilon \rightarrow 0$ expansion of the integral $A_{\epsilon}^{(1)} \quad 14$

C Analysis of the holographic result for cusp angle near zero and near $\pi \quad 15$

D Comments on the shape of the $\alpha$-dependence of $\log W_{\text {ren }} \quad 16$

\section{Introduction}

The study of ultraviolet divergences of Wilson loops due to cusps and self-intersections of their contours has a long history. It started in the early eighties [1, 2], and over the years a lot of information about the corresponding weak coupling perturbation theory has been accumulated. Of particular interest has been the cusp anomalous dimension, which is of relevance also in various other physical settings see e.g. [3] and references therein. The behaviour at strong coupling became accessible with the AdS-CFT holography [4-6] and is a subject of ongoing interest.

The cusp anomalous dimension, and therefore also the renormalised Wilson loops for cusped contours diverge, if the opening angle of the cusp tends to zero. Only recently we started the investigation of Wilson loops for contours, which have a zero opening cusp, i.e. a spike, from the very beginning $[7,8] .^{1}$

In the first paper we found a new type of ultraviolet divergence, which is proportional to the inverse of the square root of the cutoff times the jump in the curvature at the tip of the spike. The second paper is devoted to the renormalised Wilson loops, i.e. the finite pieces remaining after subtraction of UV divergences and removal of the regularisation. To have an example where analytical results can be obtained, the analysis is performed for two touching circles with opposite orientation at the touching point. In this case, besides

\footnotetext{
${ }^{1}$ The related problem for the holographic treatment of entanglement entropies has been discussed recently in $[9,10]$.
} 
the new spike divergence, no logarithmic divergence appears. Usually the subtraction of logarithmic divergences requires the introduction of a RG-scale and thus a corresponding freedom for the renormalised quantities. But now no such RG-freedom appears in this case, and the renormalised Wilson loop turned out to be equal to one, both in lowest order weak as well as strong coupling.

To motivate the study of the present paper, let us make a small detour via the renormalisation issue for local composite operators. If such composite operators are properly renormalised, their correlation functions are finite as long as the insertion points of the operators are all separated. If two such points approach each other, the corresponding correlation function develops a short distance singularity. In the coincidence limit a new composite operator is formed. Its renormalisation can be treated either by starting with the coincidence case and renormalise afterwards or by using an operator product expansion to control the short distance expansion. The renormalised version of the new composite is then defined by subtraction of the divergent short distance terms. Up to the freedom in the choice of the RG scale both procedures yield the same result.

Let us now look at the case of a renormalised Wilson loop for a path formed by consecutive passage of two touching circles. At the touching points both circles should have the same normal direction but form cusps of non-zero opening angle $\alpha$. It will diverge for $\alpha \rightarrow 0$. This is the analogue to the previous paragraph. In both cases there appear divergences in limits where a geometrical datum approaches zero, here the angle $\alpha$, there the distance between the insertion points of the operators. The task of this paper is to check, whether via this route one gets the same result for the $\alpha=0$ case as in [8]. As a welcome byproduct of this analysis we will gain the renormalised Wilson loop for two touching circles at $\alpha \neq 0$. This extends the not so large list of contours for which explicit analytical results are known. Even for the special case of contours formed with circles the known results concern the coaxial case only, see [11] and references therein.

We will treat the local supersymmetric Maldacena-Wilson loop in $\mathcal{N}=4$ SYM theory

$$
W=\frac{1}{N}\left\langle\operatorname{tr} P \exp \int\left(i A_{\mu} \dot{x}^{\mu}+|\dot{x}| \phi_{I} \theta^{I}\right) d \tau\right\rangle .
$$

The paper is organised as follows. Section 2 is devoted to the lowest order weak coupling contribution to the renormalised Wilson loop for two touching circles of different radii $R_{1}>R_{2}$ and $\alpha \neq 0$. Besides the divergence for $\alpha \rightarrow 0$ it becomes also divergent for $R_{1} \rightarrow R_{2}$. This is the reason to start with equal radii before renormalisation in section 3 . Furthermore, this section contains the discussion of the $\alpha \rightarrow 0$ behaviour for both the unequal as well as the equal radii case. In section 4 we use the holographic formula $[4,5]$

$$
\log W=-\frac{\sqrt{\lambda}}{2 \pi} A
$$

to relate the Wilson loop at strong 't Hooft coupling $\lambda=g^{2} N$ to the area $A$ of the minimal surface in AdS approaching the Wilson loop contour at its boundary. Here we will succeed only in the equal radii case, where we can make use of a suitable conformal map to the straight line cusp [6]. Section 5 is devoted to a summary and some conclusions. Several technical details of the calculations are presented in four appendices. 


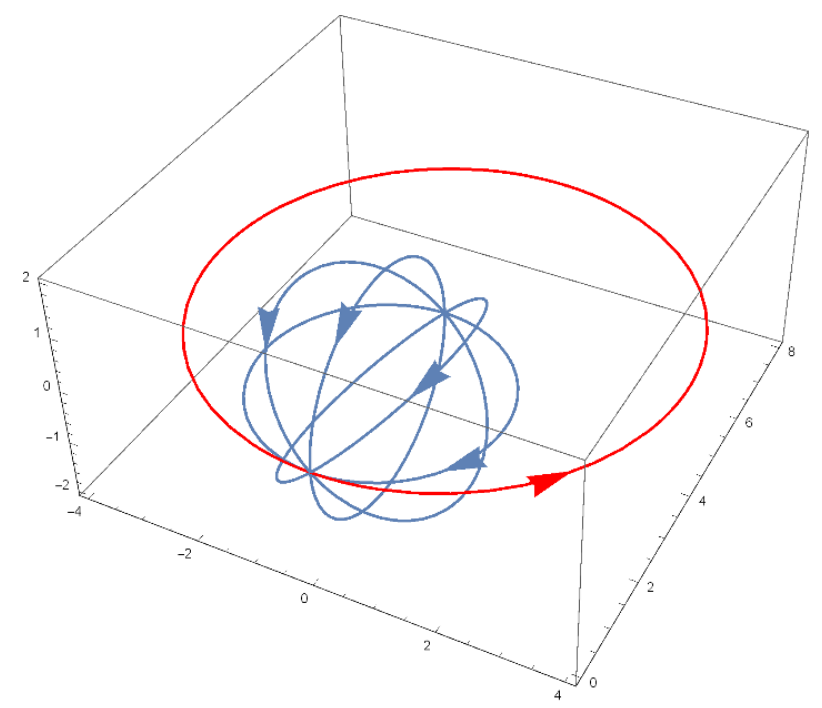

Figure 1. In red a larger circle with $R_{1}=4$. In blue smaller circles with $R_{2}=2$ for various angles $\alpha=0, \frac{\pi}{4}, \frac{\pi}{2}, \frac{3 \pi}{4}$.

\section{Lowest order at weak coupling in $\mathcal{N}=4 \mathrm{SYM}$}

We start with two circles

$$
\begin{aligned}
& \vec{x}_{1}\left(\varphi_{1}\right)=R_{1}\left(\sin \varphi_{1}, 1-\cos \varphi_{1}, 0\right), \\
& \vec{x}_{2}\left(\varphi_{2}\right)=R_{2}\left(\cos \alpha \sin \varphi_{2},\left(1-\cos \varphi_{2}\right), \sin \alpha \sin \varphi_{2}\right),
\end{aligned}
$$

with

$$
0<\alpha<\pi, \quad R_{1}>R_{2} .
$$

Then the contour to be used in (1.1) is given by

$$
\begin{aligned}
& \vec{x}(\tau)=\vec{x}_{1}(\tau), \quad 0 \leq \tau \leq 2 \pi, \\
& \vec{x}(\tau)=\vec{x}_{2}(4 \pi-\tau), \quad 2 \pi \leq \tau \leq 4 \pi .
\end{aligned}
$$

For simplicity only the case with constant $\theta_{I}$ will be considered.

For $\alpha=0$ one has the spiky situation of two touching circles with a common tangent but opposite orientation as studied in [7,8]. For $\alpha=\pi$ the circles have again a common tangent, but now with the same orientation. In the case $R_{1}=R_{2}$ the circles have two touching points. For a visualisation see figure 1. The lowest order perturbative Wilson loop has the structure ${ }^{2}$

$$
\log W=\frac{g^{2} C_{F}}{4 \pi^{2}}\left(I_{1}+I_{2}+I_{12}\right)+\mathcal{O}\left(g^{4}\right)
$$

As in $[7,8]$ we regularise the propagators by replacing $\left(\vec{x}_{1}-\vec{x}_{2}\right)^{2}$ by $\left(\vec{x}_{1}-\vec{x}_{2}\right)^{2}+\epsilon^{2}$. Then

$$
{ }^{2} C_{F}=\frac{N^{2}+1}{2 N} \text { for } \mathrm{SU}(N) \text { gauge group. }
$$


the trivial integrals $I_{j}$ are evaluated as

$$
I_{1}=I_{2}=\pi^{2}+\mathcal{O}(\epsilon)
$$

$I_{12}$ is given by

$$
I_{12}=\int_{0}^{2 \pi} \int_{0}^{2 \pi} \frac{\left(1+\cos \alpha \cos \varphi_{1} \cos \varphi_{2}+\sin \varphi_{1} \sin \varphi_{2}\right) d \varphi_{1} d \varphi_{2}}{D\left(\varphi_{1}, \varphi_{2}, \kappa, \alpha, \delta\right)}
$$

with

$$
D=2\left(\kappa\left(1-\cos \varphi_{1}\right)+\frac{1}{\kappa}\left(1-\cos \varphi_{2}\right)-\cos \alpha \sin \varphi_{1} \sin \varphi_{2}-\left(1-\cos \varphi_{1}\right)\left(1-\cos \varphi_{2}\right)\right)+\delta^{2}
$$

and the dimensionless quantities ${ }^{3}$

$$
\delta=\frac{\epsilon}{\sqrt{R_{1} R_{2}}}, \quad \kappa=\frac{R_{1}}{R_{2}}>1 .
$$

Performing the $\varphi_{2}$-integration we get

$$
I_{12}=I_{12}^{(1)}+I_{12}^{(2)},
$$

with

$$
I_{12}^{(1)}=\pi \cos \alpha \int_{0}^{2 \pi} \frac{\left(1-\frac{1}{\kappa}\right) \cos \varphi-1}{\left(1-\frac{1}{\kappa}\right)^{2}-2\left(1-\frac{1}{\kappa}\right) \cos \varphi+\cos ^{2} \alpha+\sin ^{2} \alpha \cos ^{2} \varphi} d \varphi
$$

and

$$
I_{12}^{(2)}=2 \pi \int_{0}^{2} \frac{\left(1+\cos \alpha \frac{1+\frac{\kappa \delta^{2}}{2}+\left(A+\frac{\kappa \delta^{2}}{2}(\kappa-1)\right) y+B y^{2}}{1+P y+Q y^{2}}\right) d y}{\sqrt{y(2-y)} \sqrt{\frac{\delta^{4}}{4}+\frac{\delta^{2}}{\kappa}+\left(2 \sin ^{2} \alpha+\delta^{2}(\kappa-1)\right) y+\left((\kappa-1)^{2}-\sin ^{2} \alpha\right) y^{2}}} .
$$

In the last integral we have performed the change of variables

$$
y=\left(1-\cos \varphi_{1}\right)
$$

and introduced the abbreviations

$$
\begin{array}{ll}
A=\kappa^{2}-1, & B=\kappa(\kappa-1)^{2}, \\
P=2 \kappa^{2}\left(\cos ^{2} \alpha-\frac{1}{\kappa}\right), & Q=\kappa^{2} \sin ^{2} \alpha .
\end{array}
$$

The term $I_{12}^{(1)}$ is manifestly independent of $\delta$. It turns out to be also independent of $\kappa$, equal to

$$
I_{12}^{(1)}=2 \pi^{2} \operatorname{sign}\left(\alpha-\frac{\pi}{2}\right) .
$$

The second term $I_{12}^{(2)}$ diverges if $\delta \rightarrow 0$, i.e. if the regularisation is removed.

\footnotetext{
${ }^{3}$ The limiting case of equal radii will be discussed in the next section.
} 
To control its behaviour in this limit we write

$$
I_{12}^{(2)}=I_{12}^{(2), \text { lead }}+I_{12}^{(2), \text { rest }},
$$

with

$$
I_{12}^{(2), \text { lead }}=2 \pi \int_{0}^{2} \frac{(1+\cos \alpha) d y}{\sqrt{y(2-y)} \sqrt{\frac{\delta^{2}}{\kappa}+2 y \sin ^{2} \alpha}}
$$

and $I_{12}^{(2), \text { rest }}$ defined by making in the integrand of $I_{12}^{(2)}$ the corresponding subtraction.

Now we obtain

$$
I_{12}^{(2), \text { lead }}=\frac{\pi(1+\cos \alpha)}{\sin \alpha} \log \frac{64 \kappa \sin ^{2} \alpha}{\delta^{2}}+\mathcal{O}\left(\delta^{2} \log \delta\right) .
$$

In the limit $I_{12}^{(2), \text { rest }}$ stays finite and, to extract its value, we can put $\delta=0$ under the integral. This means

$$
I_{12}^{(2), \text { rest }}=\frac{\sqrt{2} \pi}{\sin \alpha} \int_{0}^{2} \frac{d y}{y \sqrt{2-y}}\left(\frac{1+\cos \alpha \frac{1+A y+B y^{2}}{1+P y+Q y^{2}}}{\sqrt{1+S y}}-(1+\cos \alpha)\right)+\mathcal{O}\left(\delta^{2}\right),
$$

with

$$
S=\frac{(\kappa-1)^{2}-\sin ^{2} \alpha}{2 \sin ^{2} \alpha} .
$$

Some details of the evaluation of this integral one can find in appendix A. With (A.10) from that appendix and (2.17), (2.15), (2.14), (2.9) as well as (2.8) we arrive at

$$
I_{12}=-2 \pi^{2}+4 \pi \alpha+\frac{2 \pi(1+\cos \alpha)}{\sin \alpha} \log \left(\frac{8 \sin ^{2} \alpha}{\epsilon\left|\frac{1}{R_{1}}-\frac{1}{R_{2}}\right|}\right)+\mathcal{O}\left(\epsilon^{2} \log \epsilon\right) .
$$

Using this together with (2.4), (2.5) and (2.20) we get finally

$$
\log W=\frac{g^{2} C_{F}}{4 \pi^{2}}\left(4 \pi \alpha+\frac{2 \pi(1+\cos \alpha)}{\sin \alpha} \log \left(\frac{8 \sin ^{2} \alpha}{\epsilon\left|\frac{1}{R_{1}}-\frac{1}{R_{2}}\right|}\right)\right)+\mathcal{O}\left(\epsilon^{2} \log \epsilon\right) .
$$

The logarithmic divergent term $\propto \log \epsilon$ can be obtained also by studying the case of two crossing straight lines with an orientation generating two touching cusps.

As usual in situations with a logarithmic divergence, there is a renormalisation group ambiguity for defining the renormalised quantity. With a RG-scale $\mu$ we subtract the term $\propto \log (\epsilon \mu)$ and get then for the renormalised Wilson loop

$$
\log W_{\text {ren }}=\frac{g^{2} C_{F}}{4 \pi^{2}}\left(4 \pi \alpha+\frac{2 \pi(1+\cos \alpha)}{\sin \alpha} \log \left(\frac{8 \mu \sin ^{2} \alpha}{\left|\frac{1}{R_{1}}-\frac{1}{R_{2}}\right|}\right)\right)+\mathcal{O}\left(g^{4}\right) .
$$

The discussion of the $\alpha$-dependence will be postponed to the end of the next section, where it will be combined with that of the equal radii case. 


\section{The case of equal radii}

The case of equal radii $R:=R_{1}=R_{2}$, i.e. $\kappa=1$, requires separate treatment, since one is confronted with four cusps instead of two. ${ }^{4}$ The integrals $I_{1}, I_{2}$ and $I_{12}^{(1)}$ are independent of the radii and can be taken from the previous section. However, the integrand in (2.11), defining $I_{12}^{(2)}$, has now a non-integrable singularity not only at $y=0$, but also at $y=2$. Moreover, the integrand depends on $y(2-y)$ only, and we can write

$$
\left.I_{12}^{(2)}\right|_{\kappa=1}=4 \pi \int_{0}^{1} \frac{\left(1+\cos \alpha \frac{1+\frac{\delta^{2}}{2}}{1-y(2-y) \sin ^{2} \alpha}\right) d y}{\sqrt{y(2-y)} \sqrt{\frac{\delta^{4}}{4}+\delta^{2}+y(2-y) \sin ^{2} \alpha}} .
$$

Now we split

$$
\left.I_{12}^{(2)}\right|_{\kappa=1}=\left(\left.I_{12}^{(2)}\right|_{\kappa=1}\right)^{\text {lead }}+\left(\left.I_{12}^{(2)}\right|_{\kappa=1}\right)^{\text {rest }}
$$

with

$$
\left(\left.I_{12}^{(2)}\right|_{\kappa=1}\right)^{\text {lead }}=4 \pi \int_{0}^{1} \frac{(1+\cos \alpha) d y}{\sqrt{y(2-y)} \sqrt{\delta^{2}+2 y \sin ^{2} \alpha}}
$$

and $\left(\left.I_{12}^{(2)}\right|_{\kappa=1}\right)^{\text {rest }}$ defined by making in the integrand of $\left.I_{12}^{(2)}\right|_{\kappa=1}$ the corresponding subtraction. Then with manipulations similar to that in the previous section we get

$$
\begin{aligned}
& \left(\left.I_{12}^{(2)}\right|_{\kappa=1}\right)^{\text {lead }}=\frac{4 \pi(1+\cos \alpha)}{\sin \alpha} \log \left(\frac{8 \sin \alpha}{\delta \sqrt{3+2 \sqrt{2}}}\right)+\mathcal{O}\left(\delta^{2} \log \delta\right) \\
& \left(\left.I_{12}^{(2)}\right|_{\kappa=1}\right)^{\text {rest }}=\frac{4 \pi(1+\cos \alpha)}{\sin \alpha} \log \left(\frac{\sqrt{3+2 \sqrt{2}}}{2}\right)+4 \pi \alpha-4 \pi^{2} \Theta\left(\alpha-\frac{\pi}{2}\right)+\mathcal{O}\left(\delta^{2}\right) .
\end{aligned}
$$

Collecting now (2.4), (2.5), (2.8), (2.9), (2.14), (3.2), and (3.4), the final result is

$$
\left.\log W\right|_{\kappa=1}=\frac{g^{2} C_{F}}{4 \pi^{2}}\left(4 \pi \alpha+\frac{4 \pi(1+\cos \alpha)}{\sin \alpha} \log \left(\frac{4 R \sin \alpha}{\epsilon}\right)\right)+\mathcal{O}\left(\epsilon^{2} \log \epsilon\right)
$$

and

$$
\log \left(\left.W\right|_{\kappa=1}\right)_{\mathrm{ren}}=\frac{g^{2} C_{F}}{4 \pi^{2}}\left(4 \pi \alpha+\frac{4 \pi(1+\cos \alpha)}{\sin \alpha} \log (4 R \mu \sin \alpha)\right)+\mathcal{O}\left(g^{4}\right) .
$$

Comparing now this result with (2.22) we find

$$
\log W_{\text {ren }}(\alpha)=\log \left(\left.W(\alpha)\right|_{\kappa=1}\right)_{\text {ren }} \quad \text { if } \quad \frac{1}{(\mu R)^{2}}=2\left|\frac{1}{\mu R_{1}}-\frac{1}{\mu R_{2}}\right| .
$$

Therefore we comment on the $\alpha$-dependence of

$$
w(\alpha, \nu)=\frac{1}{\pi}\left(\alpha+\frac{1+\cos \alpha}{\sin \alpha} \log (4 \nu \sin \alpha)\right) .
$$

\footnotetext{
${ }^{4}$ More precise: two touching cusps and one self-intersection.
} 

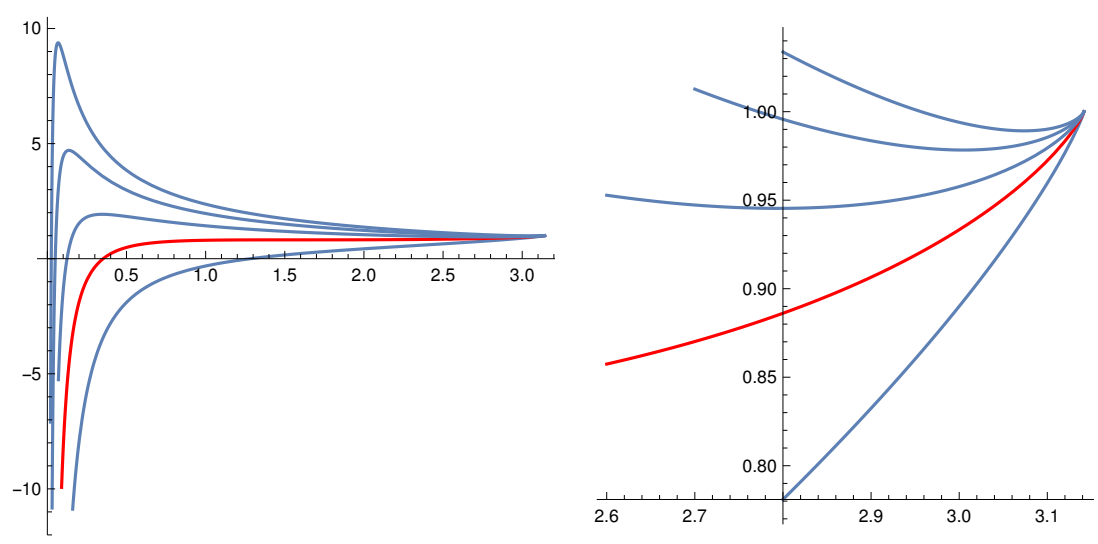

Figure 2. The function $w(\alpha, \nu)$ for $\nu=10,5,2, \frac{e}{4}, 0.1$ (from above), on the left in the whole $\alpha$-interval, on the right zoomed into the vicinity of $\alpha=\pi$. The case $\nu=\frac{e}{4}$ is shown in red.

Up to the factor $g^{2} C_{F}$ this yields (3.6) if $\nu=\mu R$ and (2.22) if $\nu=\sqrt{\frac{\mu R_{1}}{2(\kappa-1)}}$.

Now for all $\nu$

$$
w(\pi, \nu)=1, \quad w(0, \nu)=-\infty .
$$

$w(\alpha, \nu)$ is a monotonic rising function of $\alpha \in(0, \pi)$ as long as $\nu<\frac{e}{4}$. If $\nu>\frac{e}{4}$ there is a maximum $^{5}$ at $\alpha=\arcsin \frac{e}{4 \nu}$ and a local minimum at $\alpha=\pi-\arcsin \frac{e}{4 \nu}$. For some illustration see figure 2 .

\section{Holographic evaluation at strong coupling}

For generic radii $R_{1} \neq R_{2}$, we do not know of any explicit construction of the minimal surface in AdS, relevant for the holographic evaluation of our Wilson loop. However, in the special case of equal radii one can generate the two circles as the image under inversion on the unit sphere of two straight lines crossing each other at an angle $\alpha$. The intersection point has to have a distance $1 /(2 R)$ from the centre of the unit sphere, and the lines should be both orthogonal to the straight line connecting the intersection point with the centre. For a visualisation see figure 3 . The minimal surface approaching at the boundary of AdS the cusp formed by the two half-lines, with positive $x_{1}$ and $x_{2}$, is in Poincare coordinates given by [6],

$$
\begin{aligned}
& x_{1}=\rho \cos \varphi, \quad x_{2}=\rho \sin \varphi, \quad x_{3}=\frac{1}{2 R}, \\
& z=\frac{\rho}{f(\varphi)}, \quad 0 \leq \varphi \leq \alpha, \quad 0 \leq \rho<\infty .
\end{aligned}
$$

The function $f(\varphi)$ has the property

$$
f(\alpha-\varphi)=f(\varphi)
$$

and is for $0 \leq \varphi \leq \frac{\alpha}{2}$ defined implicitly by

$$
\varphi=f_{0} \sqrt{1+f_{0}^{2}} \int_{f(\varphi)}^{\infty} \frac{d f}{\sqrt{\left(f^{4}+f^{2}\right)^{2}-\left(f_{0}^{4}+f_{0}^{2}\right)\left(f^{4}+f^{2}\right)}} .
$$

\footnotetext{
${ }^{5}$ Just above $\frac{e}{4}$ only a local maximum, but soon the absolute maximum in $\alpha \in(0, \pi)$.
} 


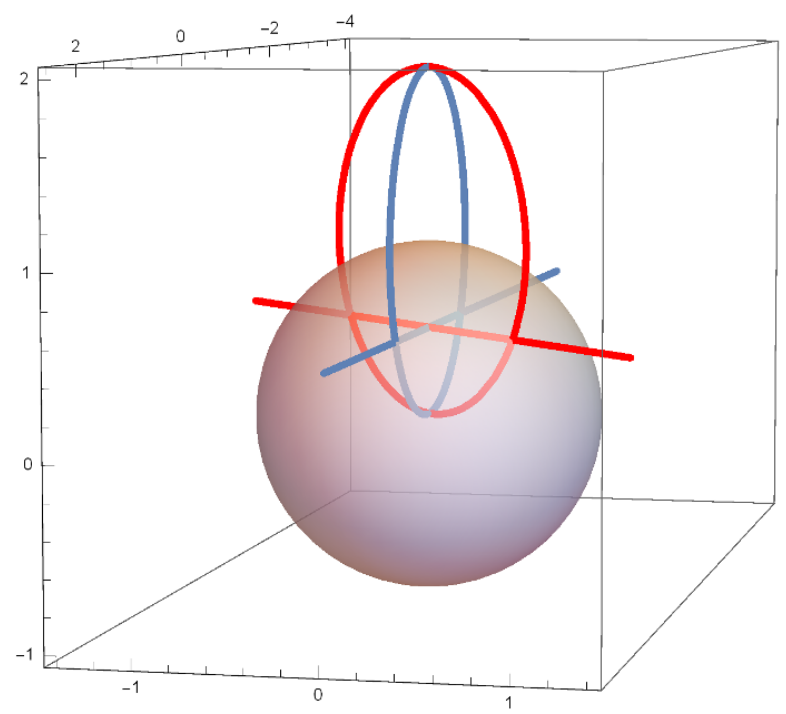

Figure 3. A transparent unit sphere with parts of the straight lines and their circular images. $R=1$ and $\alpha=\pi / 4$.

Finally, the parameter $f_{0}$ is related to the opening angle $\alpha$ by

$$
f_{0}=f\left(\frac{\alpha}{2}\right) .
$$

Extending now the inversion on the unit sphere to an isometry inside AdS, we find the part of the minimal surface related to the two half-circles with positive $x_{1}, x_{2} \cdot{ }^{6}$

$$
\begin{aligned}
x_{1} & =\frac{4 R^{2} \rho \cos \varphi}{4 R^{2} \rho^{2}\left(1+f^{-2}\right)+1}, & x_{2} & =\frac{4 R^{2} \rho \sin \varphi}{4 R^{2} \rho^{2}\left(1+f^{-2}\right)+1}, \\
x_{3} & =\frac{2 R}{4 R^{2} \rho^{2}\left(1+f^{-2}\right)+1}, & z & =\frac{4 R^{2} \rho}{f\left(4 R^{2} \rho^{2}\left(1+f^{-2}\right)+1\right)} .
\end{aligned}
$$

For a visualisation of this surface in $\mathrm{AdS}_{4}$ we have to rely on projections onto threedimensional subspaces. Before presenting corresponding figures, it is useful to take notice of the following facts.

$$
\begin{aligned}
x_{j}(\hat{\rho}, \varphi) & =x_{j}(\rho, \varphi), \quad j=1,2, \\
x_{3}(\hat{\rho}, \varphi) & =2 R-x_{3}(\rho, \varphi), \\
z(\hat{\rho}, \varphi) & =z(\rho, \varphi),
\end{aligned}
$$

with

$$
\hat{\rho}=\frac{f(\varphi)^{2}}{4 \rho R^{2}\left(1+f^{2}\right)} .
$$

Furthermore, the surface parameter point $(\rho, \varphi)$ on (4.5) has the same $x_{1}, x_{2}$ and $z$-coordinate as the parameter point $\left(\frac{4 R^{2} f^{2} \rho}{f^{2}+4 \rho^{2} R^{2}\left(1+f^{2}\right)}, \varphi\right)$ on the preimage (4.1). These analytic properties help to understand the projections obtained numerically for the case $R=1$ and $\alpha=\pi / 4$, as shown in figure 4 .

\footnotetext{
${ }^{6}$ The other part is obtained by $x_{j} \rightarrow-x_{j}, j=1,2$ in (4.5). Both parts are separated up to the two touching points of the circles.
} 

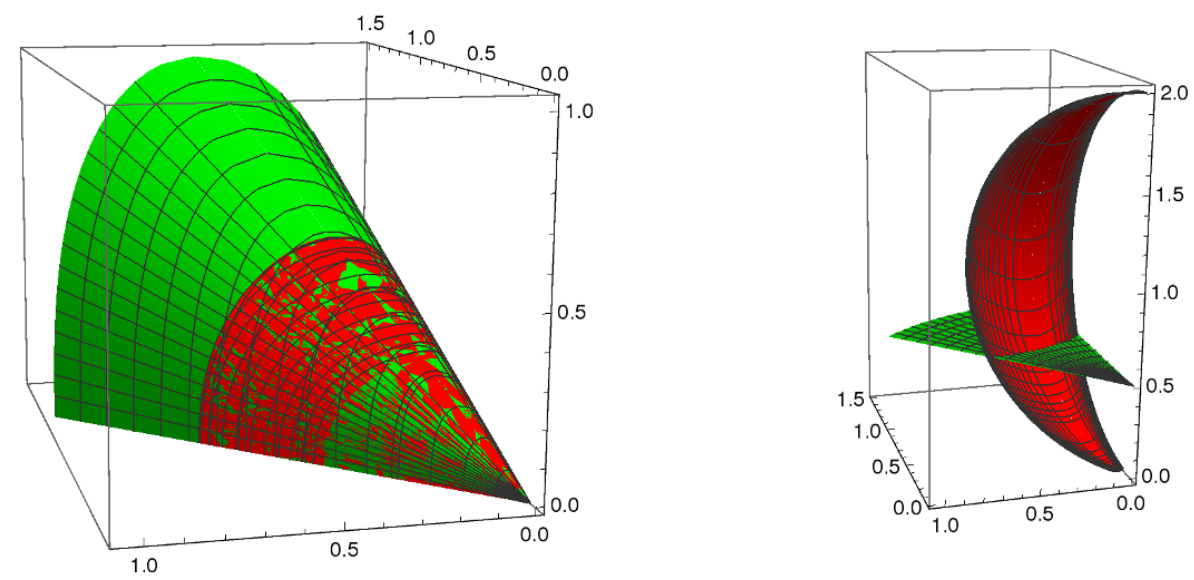

Figure 4. Projections on the $\left(x_{1}, x_{2}, z\right)$-subspace (left) and on the $\left(x_{1}, x_{2}, x_{3}\right)$-subspace (right), in red for the surface under discussion, in green for its preimage, green extends to infinity, for red the whole surface is shown, $R=1$ and $\alpha=\frac{\pi}{4}$.

The regularised area $A_{\epsilon}$, needed for the holographic evaluation of our Wilson loop, is now given by the double of the area of the surface (4.5) cutted at $z=\epsilon$. The factor two takes into account the second part, mentioned in footnote 6 . The boundary curve of the cutted surface is in surface coordinates $(\rho, \varphi)$ defined by

$$
\epsilon=\frac{4 R^{2} \rho f(\varphi)}{4 R^{2} \rho^{2}\left(1+f^{2}\right)+f^{2}} .
$$

The induced metric looks simpler on the preimage (4.1). Therefore we prefer to take advantage of the isometry property of the map between (4.1) and (4.5) and calculate on the preimage. The preimage of the boundary curve (4.8) for some values of $\epsilon$ is shown in figure 5 .

Taking the induced metric on the preimage (4.1) from [6] we get $\left(\mathcal{B}_{\epsilon}\right.$ denoting the range of $(\rho, \varphi)$ for which the r.h.s. of (4.8) is larger than $\epsilon$.)

$$
A_{\epsilon}=2 \int_{\mathcal{B}_{\epsilon}} \frac{\sqrt{f^{4}+f^{2}+\left(f^{\prime}\right)^{2}}}{\rho} d \rho d \varphi .
$$

We now change the integration variable $\varphi$ to $f$, taking into account the symmetry (4.2) and arrive at

$$
\begin{aligned}
A_{\epsilon} & =4 \int_{f_{0}}^{f_{\epsilon}} d f \int_{\rho_{\epsilon}^{-}}^{\rho_{\epsilon}^{+}} \frac{d \rho}{\rho} \sqrt{\frac{f^{4}+f^{2}}{f^{4}+f^{2}-f_{0}^{4}-f_{0}^{2}}} \\
& =4 \int_{f_{0}}^{f_{\epsilon}} U\left(f, f_{0}\right) \log \frac{\rho_{\epsilon}^{+}(f)}{\rho_{\epsilon}^{-}(f)} d f
\end{aligned}
$$

with

$$
\begin{aligned}
\rho_{\epsilon}^{ \pm}(f) & =\frac{f}{2 \epsilon\left(1+f^{2}\right)}\left(1 \pm \sqrt{1-\frac{\epsilon^{2}}{R^{2}}\left(1+f^{2}\right)}\right), \\
f_{\epsilon} & =\sqrt{\frac{R^{2}}{\epsilon^{2}}-1}, \\
U\left(f, f_{0}\right) & =\sqrt{\frac{f^{4}+f^{2}}{f^{4}+f^{2}-f_{0}^{4}-f_{0}^{2}}} .
\end{aligned}
$$




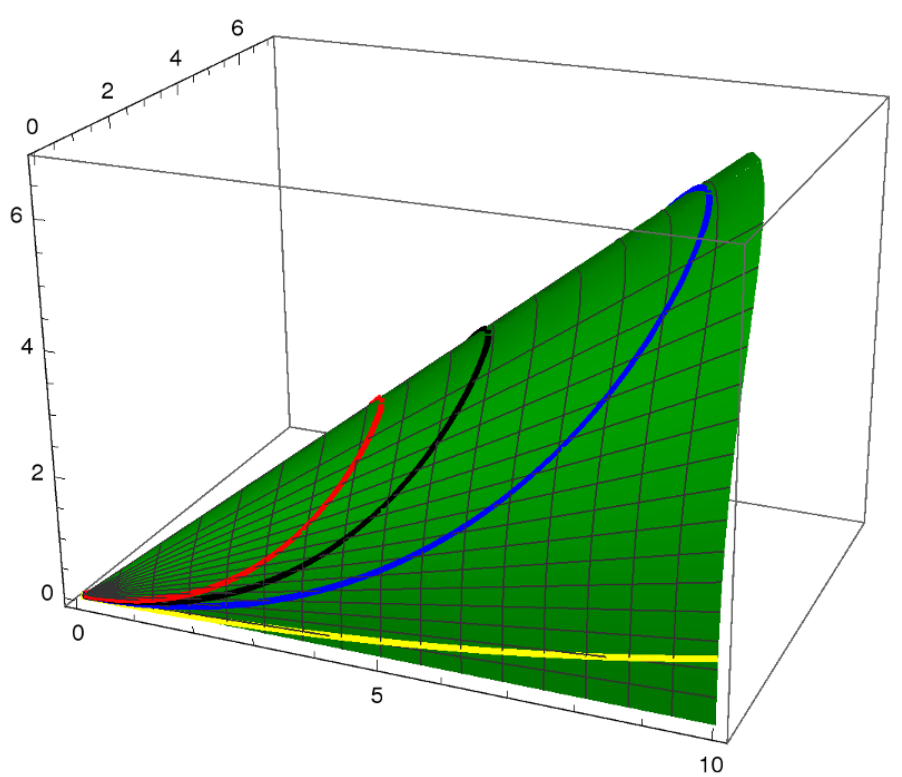

Figure 5. Preimages of the boundary of the cutted surface (4.5) for $\epsilon=0.1,0.075,0.05,0.01$ in red, black, blue, yellow. Again $R=1, \alpha=\frac{\pi}{4}$.

Using $\rho_{\epsilon}^{+} \rho_{\epsilon}^{-}=\frac{f^{2}}{4 R^{2}\left(1+f^{2}\right)}$ and again the notation $\delta=\epsilon / R$ this lead to

$$
A_{\epsilon}=A_{\epsilon}^{(1)}+A_{\epsilon}^{(2)}+A_{\epsilon}^{(3)},
$$

where the $A_{\epsilon}^{(j)}$ are given by

$$
\begin{aligned}
& A_{\epsilon}^{(1)}=8 \int_{f_{0}}^{\sqrt{\frac{1}{\delta^{2}}-1}} U\left(f, f_{0}\right) \log \left(1+\sqrt{1-\delta^{2}\left(1+f^{2}\right)}\right) d f, \\
& A_{\epsilon}^{(2)}=-8 \log \delta \int_{f_{0}}^{\sqrt{\frac{1}{\delta^{2}}-1}} U\left(f, f_{0}\right) d f \\
& A_{\epsilon}^{(3)}=-4 \int_{f_{0}}^{\sqrt{\frac{1}{\delta^{2}}-1}} U\left(f, f_{0}\right) \log \left(1+f^{2}\right) d f .
\end{aligned}
$$

Straightforward estimates yield

$$
\begin{aligned}
A_{\epsilon}^{(2)}= & 4 \Gamma_{\text {cusp }} \log \delta-8 \frac{\log \delta}{\delta}+\mathcal{O}(\delta \log \delta), \\
A_{\epsilon}^{(3)}= & 8 \frac{\log \delta}{\delta}+\frac{8}{\delta}+4 f_{0} \log \left(1+f_{0}^{2}\right)+8 \arctan f_{0}-4 \pi-8 f_{0} \\
& -4 \int_{f_{0}}^{\infty}\left(U\left(f, f_{0}\right)-1\right) \log \left(1+f^{2}\right) d f+\mathcal{O}(\delta \log \delta) .
\end{aligned}
$$

Above we introduced the strong coupling cusp anomalous dimension [6], see also [12],

$$
\begin{aligned}
\Gamma_{\text {cusp }}(\alpha) & =2 f_{0}-2 \int_{f_{0}}^{\infty}\left(U\left(f, f_{0}\right)-1\right) d f \\
& =\frac{\pi}{2} \frac{f_{0}^{2}}{\sqrt{1+f_{0}^{2}}}{ }_{2} F_{1}\left(\frac{1}{2}, \frac{3}{2}, 2, \frac{-f_{0}^{2}}{1+f_{0}^{2}}\right) .
\end{aligned}
$$



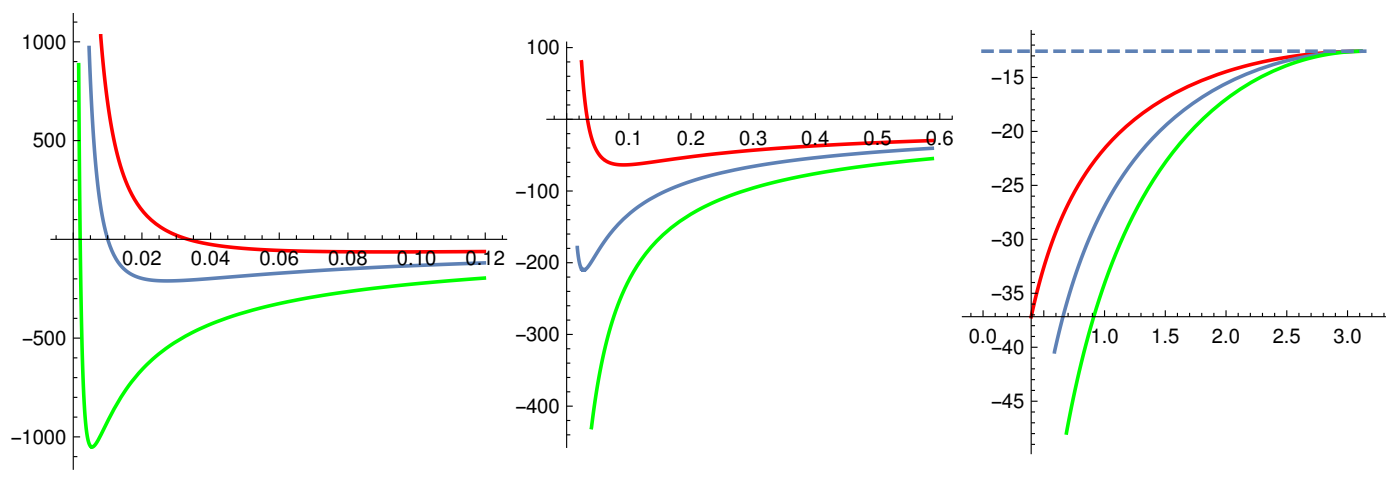

Figure 6. $A_{\text {ren }}$ as a function of $\alpha$ in different pieces of the interval $0 \leq \alpha \leq \pi$ and for $\mu R=3,10,50$ in red, blue, green. The plots are generated by ParametricPlot with $f_{0}$ as parameter. The dashed line in the right picture indicates the value $-4 \pi$.

A little bit more effort is necessary for $A_{\epsilon}^{(1)}$. It is discussed in appendix B with the result

$$
A_{\epsilon}^{(1)}=\frac{4 \pi-8}{\delta}-4 \log 2 \Gamma_{\text {cusp }}+\mathcal{O}(\sqrt{\delta})
$$

Now inserting (4.18), (4.19), (4.21) into (4.14) we arrive at

$$
A_{\epsilon}=\frac{4 \pi R}{\epsilon}-4 \Gamma_{\text {cusp }} \log \frac{2 R}{\epsilon}+A_{0}+\mathcal{O}(\sqrt{\epsilon}),
$$

with

$$
A_{0}=4 f_{0} \log \left(1+f_{0}^{2}\right)+8 \arctan f_{0}-4 \pi-8 f_{0}-4 \int_{f_{0}}^{\infty}\left(U\left(f, f_{0}\right)-1\right) \log \left(1+f^{2}\right) d f .
$$

$\Gamma_{\text {cusp }}$ and $A_{0}$ are via (4.3) and (4.4) functions of the cusp angle $\alpha$.

For the construction of a renormalised area, we again have to handle the ambiguity in the subtraction of a logarithmic divergent term by introducing a RG-scale $\mu$

$$
A_{\text {ren }}=A_{0}-4 \Gamma_{\text {cusp }} \log (2 R \mu) .
$$

A numeric evaluation for three different values of $\mu R$ can be seen in figure 6 .

For the discussion below the behaviour of $A_{\text {ren }}$ near $\alpha=0$ and $\alpha=\pi$ is of special interest. With the results of appendix C we get using (C.1), (C.4) and (C.8)

$$
A_{\text {ren }}=-4 \pi+\mathcal{O}(\pi-\alpha) .
$$

Near $\alpha=0$ we find with (C.2), (C.5), (C.6), (C.16)

$$
\begin{aligned}
A_{\text {ren }} & =-4 a b \frac{\log \alpha}{\alpha}-\left(b\left(8+a_{2}\right)+4 a b \log \frac{2 R \mu}{b}\right) \frac{1}{\alpha}+\mathcal{O}(\alpha \log \alpha) \\
& =-5.742^{\prime} \frac{\log \alpha}{\alpha}-\left(9.214^{\prime}+5.742^{\prime} \log (2 R \mu)\right) \frac{1}{\alpha}+\mathcal{O}(\alpha \log \alpha) .
\end{aligned}
$$

The strong oscillation of $A_{\text {ren }}$ in figure 6 near $\alpha=0$ is due to the fact that the leading divergent term is positive while the nextleading divergent term is negative. ${ }^{7}$

\footnotetext{
${ }^{7}$ As long as $R \mu>\frac{b}{2} \exp \left(-\frac{8+a_{2}}{4 a}\right)=0.10048 \ldots$
} 
In [12] the renormalised area of the minimal surface for a boundary contour in a plane, which is composed out of two circular arcs of radii $R_{1}, R_{2}$ forming cusps of angle $\alpha$ with a distance $D$ between the tips of the cusps, has been calculated. Comparing it with our present result for a contour built from two semi-circles with equal radius $R$ and forming cusps with the same opening angle (i.e. one half of (4.24)) one finds complete agreement since in our case $D=2 R$.

Both contours can be generated as an image under inversion on the unit sphere of a suitable placed single cusp between two straight half-lines with angle $\alpha$. Therefore, the two just compared contours can be conformally mapped to each other. Moreover, both contours are special cases of a whole set of contours with two cusps of opening angle $\alpha$ composed out of circular arcs. Due to unbroken Poincaré invariance the corresponding renormalised Wilson loops will be a function of $\mu, D, R_{1}, R_{2}, \alpha$. The only conformally invariant parameter in this set is $\alpha$. The breaking of conformal invariance is due to the presence of the cusps. Since the cusp anomalous dimension depends also in the case of curved wings on $\alpha$ only [12], one should expect that the symmetry breaking term in $A_{\text {ren }}$ or $W_{\text {ren }}$, respectively, depends only on $\mu D$ and $\alpha$. As a whole one would have a conformal covariant expression, i.e. an invariant form for a function of $\mu D$ and $\alpha$ which changes its value under conformal maps only via changes of $D$. A partial check of this conjecture is given by the comparison of the two special cases above.

The situation resembles that for lightlike polygons. There is a symmetry breaking term controlled by the anomalous conformal Ward identities and, as soon as conformal invariant parameters are available, in addition a conformal invariant remainder function [13]. In the tetragon case there is even another aspect of analogy. All tetragons can be generated by a conformal map of the lightlike straight half-line cusp [14, 15].

\section{Summary and discussion}

Concerning the motivating question posed in the introduction, our main result is the observation, that in the limit $\alpha \rightarrow 0$ in all three cases (2.22), (3.6) and (4.26) (via (1.2)) $\log W_{\text {ren }}$ beyond the diverging terms has no nonzero finite contribution. Hence the procedure $\{\alpha \neq 0$, renormalise, expand for $\alpha \rightarrow 0$, subtract divergent terms, $\alpha=0\}$ yields the same result as the procedure $\{\alpha=0$, renormalise $\}$ as used in [8].

Another common feature of all three cases is found for $\alpha \rightarrow \pi$. While $\log W_{\text {ren }}$ depends for $0<\alpha<\pi$ on the RG-scale and the radii, it becomes independent of these parameters at $\alpha=\pi$. In the latter case the cusps disappear, and one has a smooth contour at hand, the doubly wounded circle. In the weak coupling cases of sections 2 and 3 one gets then four times the result for a single circle. The renormalised minimal area (4.25) becomes twice that for a single circle. ${ }^{8}$

For large $\mu R$ both the weak and strong coupling results in sections 3 and 4 show a remarkable strong oscillation near $\alpha=0$, due to the opposite sign of the leading and nextleading term.

\footnotetext{
${ }^{8}$ This observation on the weak and strong coupling limits (note the square root in (1.2)) is consistent with the all order result $W_{\text {double }}(\lambda)=W_{\text {single }}(4 \lambda)$, see $[16,17]$.
} 
This opposite sign in the strong coupling case holds for all $\mu R$, but in the weak coupling case only for large enough $\mu R$. This is a clear indication, that the interpolation for $\log W_{\text {ren }}$ between weak and strong coupling requires a genuine function of $g^{2}, \alpha$ and $\mu R$, which cannot be factorised in a product $F\left(g^{2}\right) H(\alpha, \mu R)$. One finds in appendix D some more detailed discussion of this issue.

Further work related to the issues raised in this paper should concern the study of higher order corrections, both at weak and strong coupling. It would also be very interesting to elaborate the anomalous conformal Ward identities as acting on general polygon like contours whose edges are circular arcs. As indicted at the end of the last section this could deliver important structural information on Wilson loops for this subset of contours.

\section{Acknowledgments}

I would like to thank the Quantum Field and String Theory Group at Humboldt University for kind hospitality.

\section{A Evaluation of the integral $I_{12}^{(2), \text { rest }}$}

The indefinite integral related to (2.18) is

$$
\begin{aligned}
J(\kappa, \alpha, y)= & \frac{2 \pi}{\sin \alpha}\left(2 \cos ^{2} \frac{\alpha}{2}\left(\operatorname{arctanh} \sqrt{\frac{2}{2-y}}-\operatorname{arctanh} \sqrt{\frac{2(1+S y)}{2-y}}\right)\right. \\
& \left.+f^{+} \arctan \left(g^{+} \sqrt{\frac{1+S y}{2-y}}\right)-f^{-} \arctan \left(g^{-} \sqrt{\frac{1+S y}{2-y}}\right)\right)
\end{aligned}
$$

with

$$
\begin{aligned}
& f^{ \pm}=\cos \alpha \frac{P^{2}+2 B(P+1)+2 Q(P-1)-A(P+4 Q) \pm(2 B+A-P-2 Q) \sqrt{P^{2}-4 Q}}{\sqrt{P^{2}-4 Q} \sqrt{1+2 P+4 Q} \sqrt{2 S-4 Q+(2 S-1) P \pm(2 S+1) \sqrt{P^{2}-4 Q}}}, \\
& g^{ \pm}=\frac{\sqrt{2+4 P+8 Q}}{\sqrt{2 S-4 Q+P(2 S-1) \pm(2 S+1) \sqrt{P^{2}-4 Q}}} .
\end{aligned}
$$

These long expressions simplify tremendously after insertion of (2.13) and (2.19)

$$
\begin{aligned}
& f^{+}=\operatorname{sign}(\cos \alpha) \sin \alpha \\
& f^{-}=f^{+} \operatorname{sign}\left(\frac{(\kappa-1)^{2}}{\kappa^{2}+(\kappa-1)^{2}}-\sin ^{2} \alpha\right), \\
& g^{+}=\frac{\sqrt{2}(2 \kappa-1) \sin \alpha}{\kappa \sqrt{(\kappa-1)^{2}-\kappa^{2} \sin ^{2} \alpha}+(\kappa-1)^{2}|\cos \alpha|}, \\
& g^{-}=\frac{\sqrt{2}(2 \kappa-1) \sin \alpha}{\kappa \sqrt{(\kappa-1)^{2}-\kappa^{2} \sin ^{2} \alpha}-(\kappa-1)^{2}|\cos \alpha|} \operatorname{sign}\left(\frac{(\kappa-1)^{2}}{\kappa^{2}+(\kappa-1)^{2}}-\sin ^{2} \alpha\right) .
\end{aligned}
$$


Since the arctan-function is odd, the explicit sign-factors in (A.5) and (A.7) cancel, and we can write the second line of (A.1) as (and understanding below $g^{-}$without the sign-factor in $($ A.7))

$$
f^{+}\left(\arctan \left(g^{+} \sqrt{\frac{1+S y}{2-y}}\right)-\arctan \left(g^{-} \sqrt{\frac{1+S y}{2-y}}\right)\right) .
$$

Then with an addition theorem for the arctan-function we get

$$
\begin{aligned}
J(\kappa, \alpha, y)= & \frac{2 \pi}{\sin \alpha}\left(2 \cos ^{2} \frac{\alpha}{2}\left(\operatorname{arctanh} \sqrt{\frac{2}{2-y}}-\operatorname{arctanh} \sqrt{\frac{2(1+S y)}{2-y}}\right)\right. \\
& \left.+f^{+} n \pi+f^{+} \arctan \left(\frac{\left(g^{+}-g^{-}\right) \sqrt{\frac{1+S y}{2-y}}}{1+\frac{1+S y}{2-y} g^{+} g^{-}}\right)\right) .
\end{aligned}
$$

Here $n$ is an integer, whose value can depend on $\alpha, \kappa$ and $y$. In our integration interval the argument of the arctanh-functions in (A.8) is larger than 1. Since one is free to add an arbitrary constant in the indefinite integral we can replace in (A.8) $\operatorname{arctanh}(x)=\frac{1}{2} \log \frac{1+x}{1-x}$ by $\frac{1}{2} \log \frac{x+1}{x-1}$.

With

$$
\frac{\sqrt{2}\left(g^{+}-g^{-}\right)}{2+g^{+} g^{-}}=-\operatorname{sign}(\cos \alpha) \arctan (\tan (2 \alpha))
$$

we then get for $(2.18)$, i.e. $J(\kappa, \alpha, 2)-J(\kappa, \alpha, 0)$,

$$
I_{12}^{(2), \text { rest }}=4 \pi\left(\alpha-\pi \Theta\left(\alpha-\frac{\pi}{2}\right)-\frac{1+\cos \alpha}{2 \sin \alpha} \log \frac{\kappa-1}{\sin \alpha}\right) .
$$

The integer, left open so far in the discussion above, has been determined by comparison with the numerical evaluation of (2.18). Its presence results in the term with the unitstepfunction.

\section{B $\epsilon \rightarrow 0$ expansion of the integral $A_{\epsilon}^{(1)}$}

This appendix is devoted to the $\epsilon \rightarrow 0$ expansion of $A_{\epsilon}^{(1)}$ defined in (4.15). To start with, we write it as (remember $\delta=\epsilon / R$ )

$$
\begin{aligned}
A_{\epsilon}^{(1)} & =A_{\epsilon}^{(1,1)}+A_{\epsilon}^{(1,2)}, \\
A_{\epsilon}^{(1,1)} & =8 \int_{f_{0}}^{\frac{\sqrt{1-\delta^{2}}}{\delta}}\left(U\left(f, f_{0}\right)-1\right) \log \left(1+\sqrt{1-\delta^{2}\left(1+f^{2}\right)}\right) d f, \\
A_{\epsilon}^{(1,2)} & =8 \int_{f_{0}}^{\frac{\sqrt{1-\delta^{2}}}{\delta}} \log \left(1+\sqrt{1-\delta^{2}\left(1+f^{2}\right)}\right) d f .
\end{aligned}
$$

The indefinite integral for $A_{\epsilon}^{(1,2)}$ is a certain combination of linear, logarithmic and arctan terms. Inserting the boundaries, a straightforward expansion yields

$$
A_{\epsilon}^{(1,2)}=4 \frac{\pi-2}{\delta}-8 f_{0} \log 2+\mathcal{O}(\delta) .
$$


$A_{\epsilon}^{(1,1)}$ can be written as

$$
\begin{aligned}
A_{\epsilon}^{(1,1)}= & 8 \log 2 \int_{f_{0}}^{\frac{\sqrt{1-\delta^{2}}}{\delta}}\left(U\left(f, f_{0}\right)-1\right) d f \\
& +8 \int_{f_{0}}^{\frac{\sqrt{1-\delta^{2}}}{\delta}}\left(U\left(f, f_{0}\right)-1\right) \log \frac{1+\sqrt{1-\delta^{2}\left(1+f^{2}\right)}}{2} d f .
\end{aligned}
$$

The first term in the last equation tends to the corresponding integral extended up to infinity plus a term $\mathcal{O}(\delta)$. In the second term the small $\delta$ expansion of the log-term cannot be used uniformly in the whole integration region. Therefore, we split it in two parts, one integral over the interval $\left(f_{0}, 1 / \sqrt{\delta}\right)$ and one integral over the remainder. Then in the first part the expansion of the log-term can be used, giving a contribution $\mathcal{O}(\delta)$. For the second term we use the boundedness of the log-term in the whole integration region to establish an estimate $\mathcal{O}(\sqrt{\delta})$. Hence

$$
A_{\epsilon}^{(1,1)}=8 \log 2 \int_{f_{0}}^{\infty}\left(U\left(f, f_{0}\right)-1\right) d f+\mathcal{O}(\sqrt{\delta}) .
$$

With (B.1), (B.4) and the definition of $\Gamma_{\text {cusp }}$ in (4.20) we get equation (4.21) in the main text.

\section{Analysis of the holographic result for cusp angle near zero and near $\pi$}

Here we analyse the dependence of $\Gamma_{\text {cusp }}$ and $A_{0}$ (see (4.20) and (4.23)) on the cusp angle $\alpha$. From (4.3) and (4.4) we get

$$
\begin{array}{ll}
\alpha=\pi+\mathcal{O}\left(f_{0}\right) & \text { at } \quad f_{0} \rightarrow 0, \\
\left.\alpha=\frac{b}{f_{0}}+\mathcal{O}\left(f_{0}^{-3}\right)\right), \quad b=\frac{(2 \pi)^{\frac{3}{2}}}{\left(\Gamma\left(\frac{1}{4}\right)\right)^{2}} \quad \text { at } \quad f_{0} \rightarrow \infty .
\end{array}
$$

Therefore, to control the behaviour at the boundaries of the $\alpha$ interval $(0, \pi)$ we have to look at the behaviour at $f_{0} \rightarrow \infty$ and $f_{0} \rightarrow 0$, respectively.

With the substitution $f^{2}=f_{0}^{2}+z^{2}$ one can bring $\Gamma_{\text {cusp }}$ into the form [6]

$$
\Gamma_{\text {cusp }}=2 \int_{0}^{\infty}\left(1-\sqrt{\frac{z^{2}+1+f_{0}^{2}}{z^{2}+1+2 f_{0}^{2}}}\right) d z .
$$

From there one gets easily

$$
\Gamma_{\text {cusp }}=\frac{\pi}{2} f_{0}^{2}+\mathcal{O}\left(f_{0}^{4}\right)
$$

and

$$
\begin{aligned}
\Gamma_{\text {cusp }} & =a f_{0}+\mathcal{O}\left(f_{0}^{-1}\right), \\
a & =2 \int_{0}^{\infty}\left(1-\sqrt{\frac{1+x^{2}}{2+x^{2}}}\right) d x=2 E(-1)-\frac{\left(\Gamma\left(\frac{1}{4}\right)\right)^{2}}{2 \sqrt{2 \pi}}=1.198 \ldots
\end{aligned}
$$

Next we study the asymptotics of the integral in (4.23) (with the factor 4 included). After the same substitution as above it becomes

$$
M\left(f_{0}\right)=4 \int_{0}^{\infty}\left(\sqrt{\frac{z^{2}+1+f_{0}^{2}}{z^{2}+1+2 f_{0}^{2}}}-\sqrt{\frac{z^{2}}{z^{2}+f_{0}^{2}}}\right) \log \left(1+f_{0}^{2}+z^{2}\right) d z .
$$


This yields straightforwardly for $f_{0} \rightarrow 0$

$$
M\left(f_{0}\right)=\mathcal{O}\left(f_{0}^{2}\right) .
$$

For the other limit we write $M$ as

$$
M\left(f_{0}\right)=4 f_{0} \int_{0}^{\infty}\left(\sqrt{\frac{x^{2}+1+f_{0}^{-2}}{x^{2}+2+f_{0}^{-2}}}-\sqrt{\frac{x^{2}}{x^{2}+1}}\right) \log \left(f_{0}^{2}\left(1+x^{2}\right)+1\right) d x
$$

and get

$$
M\left(f_{0}\right)=a_{1} f_{0} \log f_{0}+a_{2} f_{0}+\mathcal{O}\left(\frac{\log f_{0}}{f_{0}}\right),
$$

with

$$
\begin{aligned}
& a_{1}=8 \int_{0}^{\infty}\left(\sqrt{\frac{x^{2}+1}{x^{2}+2}}-\sqrt{\frac{x^{2}}{x^{2}+1}}\right) d x=3.204 \ldots \\
& a_{2}=4 \int_{0}^{\infty}\left(\sqrt{\frac{x^{2}+1}{x^{2}+2}}-\sqrt{\frac{x^{2}}{x^{2}+1}}\right) \log \left(1+x^{2}\right) d x=0.556 \ldots
\end{aligned}
$$

Both constants can be also expressed in terms of standard special functions

$$
\begin{aligned}
& a_{1}=8\left(1-E(-1)+\frac{\left(\Gamma\left(\frac{1}{4}\right)\right)^{2}}{4 \sqrt{2 \pi}}\right)=8-4 a, \\
& a_{2}=\frac{(4+\pi)(2 \pi)^{\frac{3}{2}}}{\left(\Gamma\left(\frac{1}{4}\right)\right)^{2}}-8 .
\end{aligned}
$$

Inserting (C.8) and (C.10), (C.13) respectively into (4.23) we get for $f_{0} \rightarrow 0$

$$
A_{0}=-4 \pi+\mathcal{O}\left(f_{0}^{2}\right)
$$

and for $f_{0} \rightarrow \infty$

$$
A_{0}=4 a f_{0} \log f_{0}-\left(8+a_{2}\right) f_{0}+\mathcal{O}\left(\frac{\log f_{0}}{f_{0}}\right)
$$

\section{Comments on the shape of the $\alpha$-dependence of $\log W_{\text {ren }}$}

Here we add some comments comparing the shape of the $\alpha$-dependence of $\log W_{\text {ren }}$ in the equal radii case for weak and strong coupling. If there would be for all couplings a factorisation $\log W_{\text {ren }}=F\left(g^{2}\right) H(\mu R, \alpha)$, then

$$
\frac{\log W_{\text {ren }}\left(g^{2}, \mu R, \alpha\right)}{\log W_{\text {ren }}\left(g^{2}, \mu R, \pi\right)}
$$

would be an universal shape function independent of the coupling and normalised to one at $\alpha=\pi$. With (3.6) we get at weak coupling

$$
\left.\frac{\log W_{\text {ren }}\left(g^{2}, \mu R, \alpha\right)}{\log W_{\text {ren }}\left(g^{2}, \mu R, \pi\right)}\right|_{\text {weak }}=\frac{1}{\pi}\left(\alpha+\frac{1+\cos \alpha}{\sin \alpha} \log (4 \mu R \sin \alpha)\right) .
$$



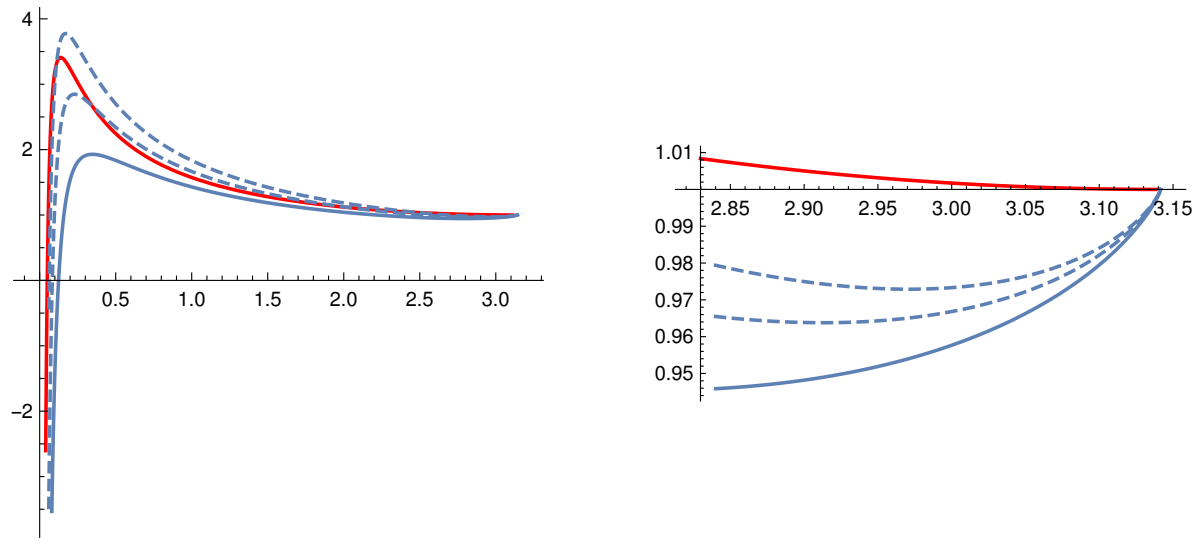

Figure 7. Comparison of the shape of the $\alpha$-dependence for strong coupling (red) and weak coupling (blue). The red and the solid blue curve are for $\mu R=2$. The blue dashed curves are for $\mu R=3$ and 4 , respectively. The right picture is a zoom into the vicinity of $\alpha=\pi$.

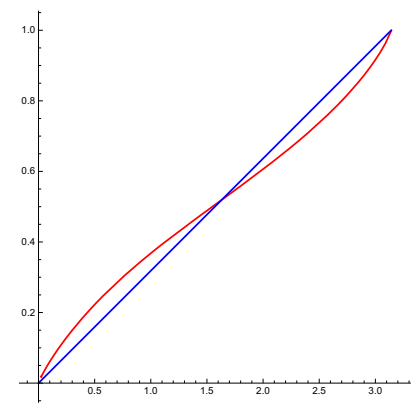

Figure 8. Comparison of the shape of the $\alpha$-dependence of candidates for a remainder function for strong coupling (red) and weak coupling (blue).

At strong coupling holds via (1.2), (4.23), (4.24), (4.25)

$$
\left.\frac{\log W_{\text {ren }}\left(g^{2}, \mu R, \alpha\right)}{\log W_{\text {ren }}\left(g^{2}, \mu R, \pi\right)}\right|_{\text {strong }}=-\frac{A_{\text {ren }}(\mu R, \alpha)}{4 \pi} .
$$

In figure 7 we show a numeric plot of both the weak and strong coupling shape functions for $\mu R=2$. They differ clearly. Obviously also by playing with different RG-scales $\mu$ no agreement can be obtained. This is another check, that there cannot exist an overall factorisation as asked for at the beginning of this appendix.

What could be possible candidates for a remainder function (and normalised to one at $\alpha=\pi$ ) in the sense of the discussion at the end of section 4? Looking at (3.6) it could be $\frac{\alpha}{\pi}$ at weak coupling, and with $(4.24),(4.23),\left(\right.$ C.16) $\frac{-A_{0}\left(f_{0}\right)+4 a f_{0} \log f_{0}-\left(8+a_{2}\right) f_{0}}{4 \pi}$ at strong coupling. We plot both functions in figure 8. A zoom into the vicinity of the crossing of both curves shows that it is located near 1.63, i.e. not at $\frac{\pi}{2}$.

Open Access. This article is distributed under the terms of the Creative Commons Attribution License (CC-BY 4.0), which permits any use, distribution and reproduction in any medium, provided the original author(s) and source are credited. 


\section{References}

[1] A.M. Polyakov, Gauge Fields as Rings of Glue, Nucl. Phys. B 164 (1980) 171 [InSPIRE].

[2] R.A. Brandt, F. Neri and M.-a. Sato, Renormalization of Loop Functions for All Loops, Phys. Rev. D 24 (1981) 879 [InSPIRE].

[3] A. Grozin, J.M. Henn, G.P. Korchemsky and P. Marquard, The three-loop cusp anomalous dimension in QCD and its supersymmetric extensions, JHEP 01 (2016) 140 [arXiv: 1510.07803] [INSPIRE].

[4] J.M. Maldacena, Wilson loops in large $N$ field theories, Phys. Rev. Lett. 80 (1998) 4859 [hep-th/9803002] [INSPIRE].

[5] S.-J. Rey and J.-T. Yee, Macroscopic strings as heavy quarks in large $N$ gauge theory and anti-de Sitter supergravity, Eur. Phys. J. C 22 (2001) 379 [hep-th/9803001] [INSPIRE].

[6] N. Drukker, D.J. Gross and H. Ooguri, Wilson loops and minimal surfaces, Phys. Rev. D 60 (1999) 125006 [hep-th/9904191] [INSPIRE].

[7] H. Dorn, On a new type of divergence for spiky Wilson loops and related entanglement entropies, JHEP 03 (2018) 124 [Erratum ibid. 05 (2018) 108] [arXiv:1801.10367] [INSPIRE].

[8] H. Dorn, On Wilson loops for two touching circles with opposite orientation, J. Phys. A 52 (2019) 095401 [arXiv: 1811.00799] [INSPIRE].

[9] P. Bueno, H. Casini and W. Witczak-Krempa, Generalizing the entanglement entropy of singular regions in conformal field theories, arXiv: 1904.11495 [INSPIRE].

[10] M. Ghasemi and S. Parvizi, Curved Corner Contribution to the Entanglement Entropy in an Anisotropic Spacetime, arXiv:1905.01675 [INSPIRE].

[11] D. Correa, P. Pisani, A. Rios Fukelman and K. Zarembo, Dyson equations for correlators of Wilson loops, JHEP 12 (2018) 100 [arXiv:1811.03552] [INSPIRE].

[12] H. Dorn, Wilson loops at strong coupling for curved contours with cusps, J. Phys. A 49 (2016) 145402 [arXiv:1509.00222] [INSPIRE].

[13] J.M. Drummond, J. Henn, G.P. Korchemsky and E. Sokatchev, Conformal Ward identities for Wilson loops and a test of the duality with gluon amplitudes, Nucl. Phys. B 826 (2010) 337 [arXiv:0712.1223] [INSPIRE].

[14] M. Kruczenski, A Note on twist two operators in $N=4 S Y M$ and Wilson loops in Minkowski signature, JHEP 12 (2002) 024 [hep-th/0210115] [INSPIRE].

[15] L.F. Alday and J.M. Maldacena, Gluon scattering amplitudes at strong coupling, JHEP 06 (2007) 064 [arXiv:0705.0303] [INSPIRE].

[16] N. Drukker and D.J. Gross, An Exact prediction of $N=4$ SUSYM theory for string theory, J. Math. Phys. 42 (2001) 2896 [hep-th/0010274] [InSPIRE].

[17] V. Pestun, Localization of gauge theory on a four-sphere and supersymmetric Wilson loops, Commun. Math. Phys. 313 (2012) 71 [arXiv:0712.2824] [INSPIRE]. 